Check for updates

Cite this: Chem. Commun., 2019 55, 13466

Received 13th September 2019, Accepted 16th October 2019

DOI: $10.1039 / c 9 c c 07168 g$

rsc.li/chemcomm

\section{On-surface synthesis of polyazulene with 2,6-connectivity $\dagger$}

\author{
Qiang Sun, (D) $\ddagger^{a}$ Ian Cheng-Yi Hou, (D) $\ddagger^{b}$ Kristjan Eimre, (D) a Carlo A. Pignedoli, (D) ${ }^{a}$ \\ Pascal Ruffieux, (D) a Akimitsu Narita (D) *bc and Roman Fasel (D) *ad
}

\begin{abstract}
Azulene, the smallest neutral nonalternant aromatic hydrocarbon, serves not only as a prototype for fundamental studies but also as a versatile building block for functional materials because of its unique opto(electronic) properties. Here, we report the on-surface synthesis and characterization of the homopolymer of azulene connected exclusively at the 2,6-positions using 2,6-diiodoazulene as the monomer precursor. As an intermediate to the formation of polyazulene, a gold-(2,6-azulenylene) chain is observed.
\end{abstract}

Properties of carbon-based aromatic systems are sensitively determined by their bond topologies. ${ }^{1}$ So far, much attention has been paid to the design and synthesis of aromatic materials like conjugated polymers and nanographenes constituted by alternant hydrocarbons, which do not possess odd-membered rings. In contrast, incorporation of non-alternant hydrocarbons has only rarely been explored. The electronic and optical properties of alternant and non-alternant hydrocarbons differ profoundly. Azulene (Scheme 1), for example, an aromatic hydrocarbon containing $10 \pi$-electrons, has several characteristics that differ from its isomer naphthalene. ${ }^{2}$ Azulene has an intrinsic dipole moment of $1.08 \mathrm{D},{ }^{3}$ while naphthalene is non-polar. The dipole moment of azulene arises from an unequal distribution of electron density between its electron-deficient 7-membered ring and electron-rich 5-membered ring due to an aromatic stabilization according to Hückel's rule. In addition, azulene exhibits "anomalous" fluorescence in its second excited singlet state in violation

\footnotetext{
${ }^{a}$ Empa, Swiss Federal Laboratories for Materials Science and Technology, Überlandstrasse 129, CH-8600 Dübendorf, Switzerland.

E-mail:Roman.Fasel@empa.ch

${ }^{b}$ Max Planck Institute for Polymer Research, 55128 Mainz, Germany. E-mail:narita@mpip-mainz.mpg.de

${ }^{c}$ Organic and Carbon Nanomaterials Unit, Okinawa Institute of Science and Technology Graduate University, 1919-1 Tancha, Onna-son, Kunigami, Okinawa 904-0495, Japan

${ }^{d}$ Department of Chemistry and Biochemistry, University of Bern, Freiestrasse 3, CH-3012 Bern, Switzerland

$\dagger$ Electronic supplementary information (ESI) available. See DOI: 10.1039/c9cc07168g

\$ These authors contributed equally to this work.
}

a

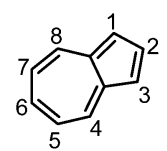

b

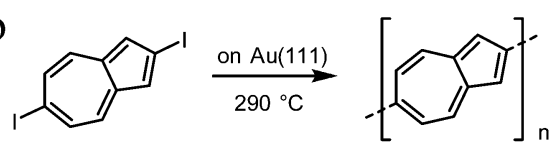

Scheme 1 (a) The chemical structure of azulene with carbon atom numbering. (b) On-surface synthetic route toward 2,6-polyazulene.

of Kasha's rule, ${ }^{4}$ which makes it a promising candidate in optoelectronics. ${ }^{5}$

Because of its unique electronic and optical properties, azulene has been employed as a core structure of functional materials for different applications such as stimuli-responsive materials, ${ }^{6}$ organic field-effect transistors, ${ }^{7}$ solar cells ${ }^{8}$ and others. ${ }^{9}$ The connectivity of azulenylene units (Scheme 1a) in the derived structures has a substantial influence on their optical and electronic properties. ${ }^{10}$ One remarkable example is that the lowest unoccupied molecular orbital (LUMO) of $2,6^{\prime}: 2^{\prime}, 6^{\prime \prime}$-terazulene is fully delocalized over the whole molecule, showing strong bonding between the azulene moieties, resulting in a good n-type semiconductor performance. ${ }^{11}$ This motivates the study of 2,6-polyazulene. However, despite a number of theoretical studies on 2,6-polyazulenes, reports on the synthesis of azulene-based polymers predominantly focus on the incorporation of 1,3-azulenylenes. ${ }^{12}$ Recent studies showed that integration of 2,6-azulenylenes into copolymers has great potential for field-effect transistors and proton-conducting materials. ${ }^{7 b, 13}$ Nevertheless, the synthesis of azulene homopolymers with 2,6-connectivity remains elusive.

On-surface synthesis has recently developed as a complementary strategy for chemical synthesis. A significant number of structures/materials which are challenging by conventional solution chemistry have been obtained by this method. ${ }^{14}$ Due to its potentially interesting properties, 2,6-polyazulene is starting to gain attention from the community of on-surface synthesis. ${ }^{15}$ Here, we report the on-surface synthesis of polyazulene with exclusive 2,6-connectivity using 2,6-diiodoazulene as the molecular precursor (Scheme 1b). The electronic properties of the synthesized 
polyazulene are investigated by scanning tunneling spectroscopy (STS) and density functional theory (DFT) calculations. The electronic gap of the polyazulene is determined to be $1.8 \mathrm{eV}$ on $\mathrm{Au}(111)$, which is further supported by differential conductance $\mathrm{d} I / \mathrm{d} V$ mapping. This work demonstrates not only the synthesis but also the first detailed electronic characterization of azulene homopolymers with pure 2,6-connectivity.

2,6-Diiodoazulene was synthesized following a previous work. ${ }^{16}$ To access the pristine 2,6-diiodoazulene molecules, we first deposit the molecule under ultrahigh vacuum conditions onto an $\mathrm{Au}(111)$ surface maintained at $160 \mathrm{~K}$ to avoid possible deiodination that would occur at room temperature. ${ }^{17}$ As shown in the scanning tunneling microscopy (STM) images (Fig. 1a and b), 2,6diiodoazulene forms porous structures on $\mathrm{Au}(111)$. A zoom-in image reveals the monomers appearing as rod-shaped structures with brighter iodine protrusions at both ends according to the chemical model displayed in Fig. 1b. Note that it is not possible to differentiate the 7- and 5-membered rings and the exact orientation of azulenes solely based on the STM images. After annealing the

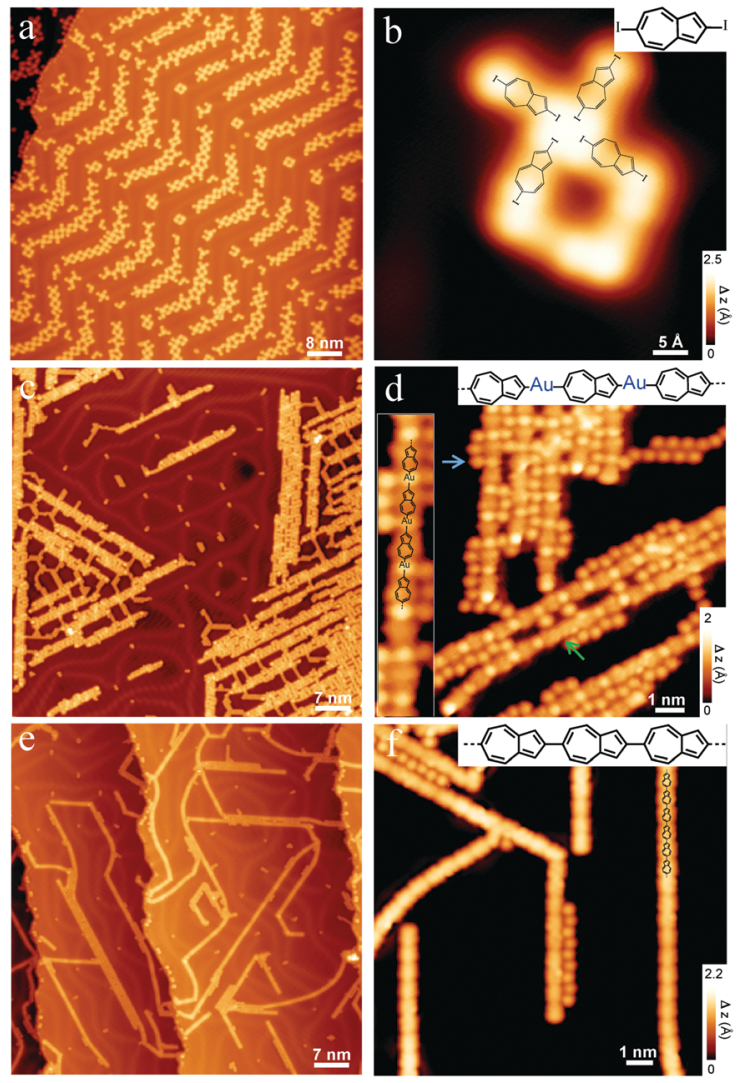

Fig. 1 (a and b) STM images after deposition of 2,6-diiodoazulene on $\mathrm{Au}(111)$ held at $160 \mathrm{~K}$. Chemical structures of four molecules are overlaid on the corresponding STM image in (b). (c and d) STM images of the sample after annealing to $350 \mathrm{~K}$. The inset in (d) highlights the organo-metallic chain. The blue arrow indicates a detached iodine atom. The green arrow indicates oligomeric 2,6-connected azulene segments. (e and f) STM images after annealing the sample to $580 \mathrm{~K}$. A scaled chemical model of a 2,6oligoazulene is overlaid on a chain in (f). Scanning parameters: (a) $V_{\mathrm{s}}=-1 \mathrm{~V}$, $I_{\mathrm{t}}=0.09 \mathrm{nA}$; (b) $V_{\mathrm{s}}=-0.02 \mathrm{~V}, I_{\mathrm{t}}=0.25 \mathrm{nA}$; (c) $V_{\mathrm{s}}=-1 \mathrm{~V}, I_{\mathrm{t}}=0.06 \mathrm{nA}$; (d) $V_{\mathrm{s}}=$ $-0.005 \mathrm{~V}, I_{\mathrm{t}}=0.1 \mathrm{nA}$; (e) $V_{\mathrm{s}}=-1 \mathrm{~V}, I_{\mathrm{t}}=0.08 \mathrm{nA}$; (f) $V_{\mathrm{s}}=-0.01 \mathrm{~V}, I_{\mathrm{t}}=1 \mathrm{nA}$. sample to $350 \mathrm{~K}$, a chain structure is observed on the surface (Fig. 1c and d). The molecular chains are composed of two alternating subunits (Fig. 1d), assigned to 2,6-azulenylene moieties and to gold adatoms, respectively. We superimpose an equally scaled chemical structure of a chain segment onto a small-scale STM image in Fig. 1d, which shows a good match. It is not surprising that gold atoms are involved in nanostructures obtained upon dehalogenation of molecules on Au substrates, and there are many reports on organo-metallic species on $\mathrm{Au}(111) .{ }^{18}$ The detached iodine atoms are detected as round protrusions staying aside the organo-metallic chains (indicated by a blue arrow in Fig. 1d), forming some honeycomb-type structure as shown on the left side of Fig. 1c. This is consistent with the fact that iodine desorption from $\mathrm{Au}(111)$ starts only at around $540 \mathrm{~K} .{ }^{17}$ Closer inspection of the structures formed at $350 \mathrm{~K}$ reveals that a few 2,6-connected oligoazulenes without the involvement of $\mathrm{Au}$ atoms have already been formed (one of them is indicated by a green arrow in Fig. 1d). The one-dimensional organometallic chains are straight due to the formation of the $\mathrm{C}-\mathrm{Au}-\mathrm{C}$ organo-metallic motifs as aryl radicals on $\mathrm{Au}(111) .{ }^{18}$ Further annealing the sample to $580 \mathrm{~K}$ triggers the release of all $\mathrm{Au}$ atoms from the organo-metallic chains and the formation of the carbon-carbon bonded polymer, as well as partial desorption of iodines (Fig. 1e and f). A high-resolution STM image of the polymer reveals that the azulene units are connected along their long axis, implying the formation of 2,6-connected polyazulene.

To further support the formation of polyazulene and determine the connectivity between azulene units, we resort to bond-resolved non-contact atomic force microscopy (nc-AFM). ${ }^{19}$ As shown in Fig. 2a, the structure of the polymer can be clearly resolved, with the 5- and 7-membered rings being imaged with different sizes. Typical defects in the straight 2,6-connected polyazulene are kinks (cf. Fig. S1, ESI $\dagger$ ) arising from 1,6-connected azulene units, which are attributed to a small amount of impurity from the precursor ${ }^{16}$ or to a 2,1-sigmatropic rearrangement. Within a 2,6-polyazulene chain, the neighboring 2,6-azulenylenes can be connected in three different ways, namely pentagon $v s$. heptagon $(\mathrm{p}-\mathrm{h})$, pentagon $v s$. pentagon (p-p), or heptagon vs. heptagon (h-h), which correspond to 2,6-,
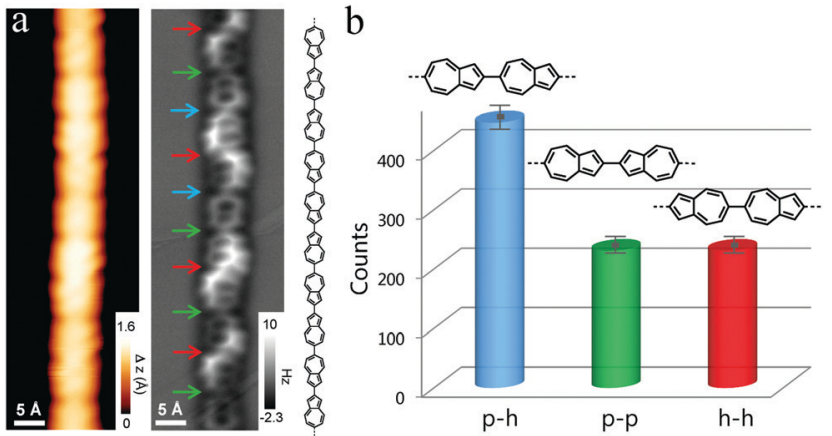

Fig. 2 (a) High-resolution STM image of a 2,6-polyazulene chain $\left(V_{s}=\right.$ $\left.-0.1 \mathrm{~V}, I_{\mathrm{t}}=0.3 \mathrm{nA}\right)$ and the corresponding nc-AFM image $\left(V_{\mathrm{s}}=-5 \mathrm{mV}\right.$, Oscillation amplitude: $\sim 80 \mathrm{pm})$. The pentagon-heptagon $(\mathrm{p}-\mathrm{h})$, pentagonpentagon $(p-p)$ and heptagon-heptagon $(h-h)$ linkages are indicated by blue, green and red arrows, respectively. The corresponding chemical model of the chain is also shown. (b) Statistical analysis of the different linkages within the polymers. 
2,2- and 6,6-connectivity, respectively (see the arrows in Fig. 2a). The h-h-connectivity is the most easily recognized, since it results in a non-zero dihedral angle between the neighboring azulenylene units due to the steric hindrance between the hydrogen atoms at 5,7-positions of azulenylene, which is clearly reflected in the nc-AFM images. High resolution images however also allow identification of $\mathrm{p}-\mathrm{h}$ - and $\mathrm{p}$-p-connectivity. An interesting aspect about the reaction between azulenylenes is whether there is any preference for the $\mathrm{p}-\mathrm{h}$-connectivity, since azulene has an intrinsic dipole moment of $1.08 \mathrm{D}$ which might direct the polymerization process via dipole-dipole interactions. To this end, we have performed a statistical analysis of the linkages between the neighboring azulenylenes. It turns out that the ratio between $\mathrm{p}-\mathrm{h}-\mathrm{p}$-p- and $\mathrm{h}$-h-linkages is $2: 1: 1$ (Fig. 2b), which indicates a non-selective, random connection between the 5- and 7-membered rings (see discussion in Fig. S2, ESI $\dagger$ ). Thus, the intrinsic dipole moment of pristine azulene does not play a role in orienting the azulenylenes during on-surface polymerization. We attribute this to the screening effect of the electrons of the metal which produces an image dipole, with the resulting dipolar interaction being inconsequentially small for molecular dipoles oriented parallel to the surface. ${ }^{20} \mathrm{~A}$ possible way to align the azulenylenes and to obtain only $\mathrm{p}-\mathrm{h}$ linkages may thus be through the use of an insulating substrate.

To study the electronic properties of 2,6-polyazulene, we first carry out DFT calculations of an azulene molecule and polyazulene. Fig. 3a shows the frontier orbitals from HOMO-2 to LUMO+1 of azulene, and Fig. $3 \mathrm{~b}$ shows the band structure of the 2,6polyazulene originating from these orbitals. The bands originating from the highest occupied molecular orbital (HOMO), HOMO-2 and LUMO+1 are relatively flat due to the orbitals having low amplitudes at the 2,6-positions where azulene units are connected. In contrast, the bands originating from HOMO-1 and LUMO show significant dispersion due to a considerable orbital overlap between the neighboring azulenylenes. To further investigate how the random distribution of $\mathrm{p}-\mathrm{h}-, \mathrm{p}-\mathrm{p}$ - and $\mathrm{h}$-h-linkages affects the electronic structure, we perform band structure calculations for three different structures consisting of a supercell with four azulene units. The first structure with only the $\mathrm{p}$-h-linkage between all the azulenylenes reproduces the band structure of 2,6-polyazulene (Fig. 3c), but folded to a four times smaller Brillouin zone. The second structure (star) in Fig. $3 c$ has alternating $\mathrm{p}-\mathrm{p}-$ and $\mathrm{h}-\mathrm{h}-$ linkages, and the third structure (diamond) has every third unit flipped compared to the others. Besides a splitting of the bands due to lifting of degeneracy near $k$-vectors corresponding to the modified periodicity, the band structures near the valence band (VB) and conduction band (CB) onsets do not differ much from each other. All of the three structures have an electronic band gap of $0.94 \pm$ $0.03 \mathrm{eV}$, and their frontier bands display similar dispersions. This is in line with the fact that the molecular orbitals of azulene possess no weight at 2,6-positions for HOMO-2 and HOMO but considerable weight for HOMO-1 and LUMO (Fig. 3a). Although three different linkages are randomly distributed within our experimentally obtained polymers, the electronic properties of the synthesized polyazulene shall remain very similar to the ones of the perfectly regular poly(2,6-azulenylene).

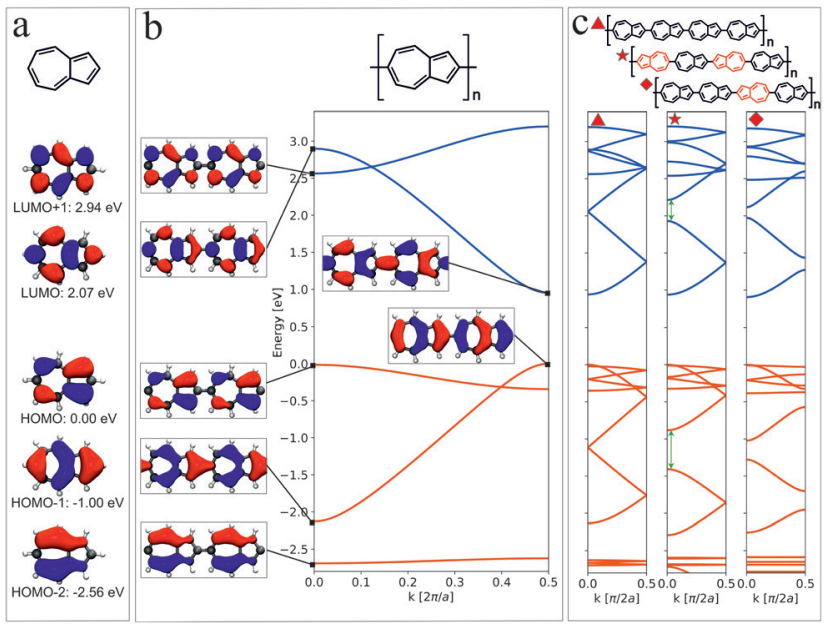

Fig. 3 Electronic structure of azulene and polyazulenes with 2,6-connectivity. (a) The shape of frontier orbitals of an azulene molecule. (b) Band structure of 2,6-polyazulene and the orbital shapes of the frontier bands which evolve from the corresponding molecular orbital of azulene shown in (a). (c) Band structures of three polyazulenes with different connectivity patterns in a supercell containing four 2,6-azulenylenes with all units oriented in the same way (triangle); first and third unit flipped (star); and only third unit flipped (rhombus). The green arrows show two examples of degeneracy liftings caused by the flipped units. In all band structure plots, the top of the valence band is taken as zero energy, the orange lines are occupied bands while the blue lines correspond to the unoccupied bands. In all orbital plots wavefunction isosurfaces at $\pm 0.03 \AA^{-3 / 2}$ are shown. $a$ is the length of one azulene unit within the homopolymer.

We have used $\mathrm{d} I / \mathrm{d} V$ spectroscopy to experimentally characterize the electronic properties. The point spectra of the polymer reveal two peaks at around $-1.25 \mathrm{~V}$ and $0.6 \mathrm{~V}$, which correspond to its VB and CB onsets, respectively (Fig. 4a and b). To verify the assignment of the molecular orbitals, we have performed $\mathrm{d} I / \mathrm{d} V$ mapping at two corresponding bias voltages (Fig. 4c). The $\mathrm{d} I / \mathrm{d} V$ maps show clear patterns confirming their origin from molecular orbitals. Moreover, the position of the $\mathrm{h}$-h-linkage has a higher contrast in $\mathrm{dI} / \mathrm{dV}$ mapping of the $\mathrm{CB}$ onset, which is highlighted by the white rectangle in Fig. 4. This feature could also be seen in the constant-current STM image at a bias voltage of $0.6 \mathrm{~V}$ (Fig. S3, ESI $\dagger$ ), and is consistent with the fact that the LUMO of an azulene monomer is mainly located at the 7-membered ring (see also Fig. 3a). ${ }^{12 a}$ To further support our experimental findings, we determine the electronic properties of the experimentally observed polyazulene segment shown in Fig. 4a by DFT calculations (Fig. S4, ESI $\dagger$ ). The DFT calculated local density of states (LDOS) maps at the VB and CB onsets (i.e., HOMO and LUMO) are in good agreement with the experimental $\mathrm{d} I / \mathrm{d} V$ maps at both negative and positive biases (Fig. 4c), and thus confirm that the peaks at $-1.25 \mathrm{~V}$ and $0.6 \mathrm{~V}$ in Fig. $4 \mathrm{~b}$ originate from the VB and CB onsets. Note that the energy positions of the DFT computed LDOS are determined from the calculated DOS shown in Fig. S4 (ESI $\dagger$ ). Notably, the h-h-linkage displays characteristic contrast in both DFT calculated maps, and appears particularly bright in the experimental map (highlighted by dashed rectangles).

The frontier states (i.e., the first occupied and unoccupied states) of the polymer are positioned symmetrically with respect 


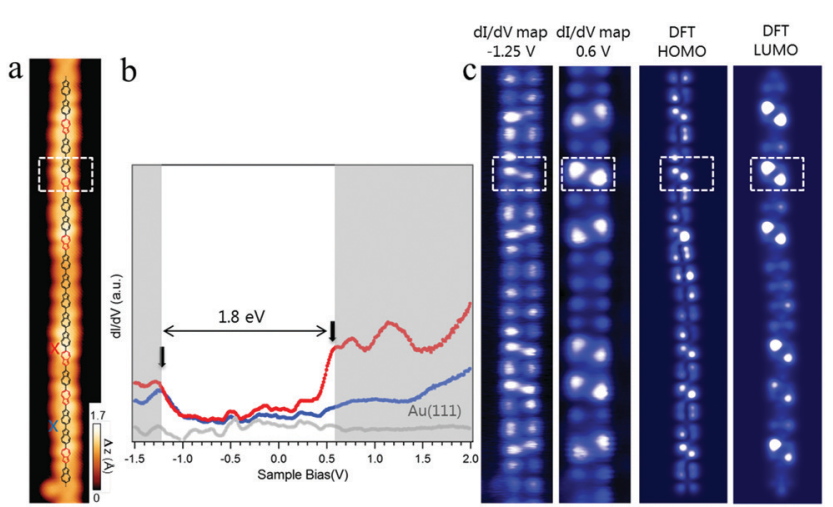

Fig. 4 (a) STM image of a polyazulene segment $\left(V_{s}=-1.5 \mathrm{~V}, I_{t}=0.9 \mathrm{nA}\right)$ and (b) its differential conductance $\mathrm{d} / / \mathrm{d} V$ spectra. The spectra were taken at the locations marked by crosses with corresponding colors in (a). The grey spectrum has been obtained on a clean $A u(111)$ area nearby. Spectra are vertically offset for clarity. (c) Constant-current $d / / d V$ maps of the HOMO and LUMO, and the corresponding DFT-calculated LDOS maps. The $\mathrm{h}$-h-linkage which shows a higher contrast is highlighted by dashed rectangles in both $\mathrm{d} / / \mathrm{d} V$ and LDOS maps.

to a negative bias voltage of around $-0.3 \mathrm{~V}$ (Fig. $4 \mathrm{~b}$ ). In contrast, the frontier states of most carbon nanowires/ribbons composed of alternant (poly)benzenoids are positioned symmetrically to positive bias voltages on $\mathrm{Au}(111) .{ }^{21}$ This is due to the systems having different valence and conduction band alignments with respect to the vacuum level, which determines the corresponding positioning with respect to the Au Fermi level upon adsorption for weakly interacting systems. This is reflected in our DFT calculations, which demonstrate that for polyazulene the $\mathrm{VB}$ and $\mathrm{CB}$ onsets are respectively at $-5.0 \mathrm{eV}$ and $-4.1 \mathrm{eV}$ with respect to the vacuum level. In contrast, the band onsets for armchair graphene nanoribbons of width 7 (7AGNR) are found at $-4.7 \mathrm{eV}$ and $-3.2 \mathrm{eV}$ (Fig. S5, ESI $\dagger$ ).

In conclusion, the homopolymer of azulene with exclusive 2,6-connectivity has been synthesized by dehalogenative coupling of 2,6-diiodoazulene on $\mathrm{Au}(111)$. Although azulene has an intrinsic dipole moment, dipole-dipole interactions do not yield a preferential pentagon-heptagon-linkage along the polymer. However, the electronic properties of 2,6-polyazulene do not significantly depend on the ratio of the $\mathrm{p}-\mathrm{p}, \mathrm{p}-\mathrm{h}$ and $\mathrm{h}-\mathrm{h}$ linkages. STS yields an electronic gap of $1.8 \mathrm{eV}$ for polyazulene on $\mathrm{Au}(111)$, confirmed by STS mapping and DFT-based LDOS simulations. The reported synthesis route may enable future applications of azulene-based functional materials.

This work was supported by the Swiss National Science Foundation under Grant No. 200020_182015, the NCCR MARVEL funded by the Swiss National Science Foundation (51NF40-182892), the European Union's Horizon 2020 research and innovation programme under grant agreement number 785219 (Graphene Flagship Core 2), the Office of Naval Research (N00014-18-1-2708), and a grant from the Swiss National Supercomputing Centre (CSCS) under project ID s904. Q. S. acknowledges the EMPAPOSTDOCS-II programme under the Marie Sklodowska-Curie grant agreement no.
754364. Open Access funding provided by the Max Planck Society. R. F. thanks Oliver Gröning for insightful discussions.

\section{Conflicts of interest}

There are no conflicts to declare.

\section{Notes and references}

1 (a) B. P. Klein, N. J. van der Heijden, S. R. Kachel, M. Franke, C. K. Krug, K. K. Greulich, L. Ruppenthal, P. Müller, P. Rosenow, S. Parhizkar, F. C. Bocquet, M. Schmid, W. Hieringer, R. J. Maurer, R. Tonner, C. Kumpf, I. Swart and J. M. Gottfried, Phys. Rev. X, 2019, 9, 011030; (b) S. Mishra, T. G. Lohr, C. A. Pignedoli, J. Liu, R. Berger, J. I. Urgel, K. Müllen, X. Feng, P. Ruffieux and R. Fasel, ACS Nano, 2018, 12, 11917-11927.

2 (a) J. Michl and E. W. Thulstrup, Tetrahedron, 1976, 32, 205-209; (b) J. W. Sidman and D. S. McClure, J. Chem. Phys., 1956, 24, 757-763. 3 A. G. Anderson Jr and B. M. Steckler, J. Am. Chem. Soc., 1959, 81, 4941-4946.

4 Y. Zhou, G. Baryshnikov, X. Li, M. Zhu, H. AAgren and L. Zhu, Chem. Mater., 2018, 30, 8008-8016.

5 (a) N. Tétreault, R. S. Muthyala, R. S. H. Liu and R. P. Steer, J. Phys. Chem. A, 1999, 103, 2524-2531; (b) D. R. Mitchell and G. D. Gillispie, J. Phys. Chem., 1989, 93, 4390-4393.

6 M. Murai, E. Amir, R. J. Amir and C. J. Hawker, Chem. Sci., 2012, 3, 2721-2725.

7 (a) Y. Yamaguchi, K. Ogawa, K. Nakayama, Y. Ohba and H. Katagiri, J. Am. Chem. Soc., 2013, 135, 19095-19098; (b) H. Xin, C. Ge, X. Jiao, X. Yang, K. Rundel, C. R. McNeill and X. Gao, Angew. Chem., 2018, 130, 1336-1340.

8 H. Nishimura, N. Ishida, A. Shimazaki, A. Wakamiya, A. Saeki, L. T. Scott and Y. Murata, J. Am. Chem. Soc., 2015, 137, 15656-15659.

9 (a) J.-X. Dong and H.-L. Zhang, Chin. Chem. Lett., 2016, 27, 1097-1104; (b) H. Xin and X. Gao, ChemPlusChem, 2017, 82, 945-956.

10 E. Amir, M. Murai, R. J. Amir, J. S. Cowart, M. L. Chabinyc and C. J. Hawker, Chem. Sci., 2014, 5, 4483-4489.

11 Y. Yamaguchi, M. Takubo, K. Ogawa, K. Nakayama, T. Koganezawa and H. Katagiri, J. Am. Chem. Soc., 2016, 138, 11335-11343.

12 (a) G. Nöll, C. Lambert, M. Lynch, M. Porsch and J. Daub, J. Phys. Chem. C, 2008, 112, 2156-2164; (b) R. P. Steer, J. Photochem. Photobiol., C, 2019, 40, 68-80.

13 I. C.-Y. Hou, V. Shetti, S.-L. Huang, K.-L. Liu, C.-Y. Chao, S.-C. Lin, Y.-J. Lin, L.-Y. Chen and T.-Y. Luh, Org. Chem. Front., 2017, 4, 773-778.

14 (a) J. Björk and F. Hanke, Chem. - Eur. J., 2014, 20, 928-934; (b) P. A. Held, H. Fuchs and A. Studer, Chem. - Eur. J., 2017, 23, 5874-5892; (c) Q. Sun, R. Zhang, J. Qiu, R. Liu and W. Xu, Adv. Mater., 2018, 30, 1705630.

15 Q. Fan, D. Martin-Jimenez, D. Ebeling, C. K. Krug, L. Brechmann, C. Kohlmeyer, G. Hilt, W. Hieringer, A. Schirmeisen and M. Gottfried, J. Am. Chem. Soc., 2019, DOI: 10.1021/jacs.9b08060.

16 M. Narita, T. Murafuji, S. Yamashita, M. Fujinaga, K. Hiyama, Y. Oka, F. Tani, S. Kamijo and K. Ishiguro, J. Org. Chem., 2018, 83, 1298-1303.

17 M. Di Giovannantonio, O. Deniz, J. I. Urgel, R. Widmer, T. Dienel, S. Stolz, C. Sánchez-Sánchez, M. Muntwiler, T. Dumslaff, R. Berger, A. Narita, X. Feng, K. Müllen, P. Ruffieux and R. Fasel, ACS Nano, 2018, 12, 74-81.

18 (a) J. I. Urgel, H. Hayashi, M. Di Giovannantonio, C. A. Pignedoli, S. Mishra, O. Deniz, M. Yamashita, T. Dienel, P. Ruffieux, H. Yamada and R. Fasel, J. Am. Chem. Soc., 2017, 139, 11658-11661; (b) H. Zhang, H. Lin, K. Sun, L. Chen, Y. Zagranyarski, N. Aghdassi, S. Duhm, Q. Li, D. Zhong, Y. Li, K. Müllen, H. Fuchs and L. Chi, J. Am. Chem. Soc., 2015, 137, 4022-4025; (c) Q. Sun, L. Cai, H. Ma, C. Yuan and W. Xu, ACS Nano, 2016, 10, 7023-7030.

19 L. Gross, F. Mohn, N. Moll, P. Liljeroth and G. Meyer, Science, 2009, 325, 1110-1114.

20 D. A. Kunkel, J. Hooper, S. Simpson, D. P. Miller, L. Routaboul, P. Braunstein, B. Doudin, S. Beniwal, P. Dowben, R. Skomski, E. Zurek and A. Enders, J. Chem. Phys., 2015, 142, 101921.

21 P. Ruffieux, J. Cai, N. C. Plumb, L. Patthey, D. Prezzi, A. Ferretti, E. Molinari, X. Feng, K. Müllen, C. A. Pignedoli and R. Fasel, ACS Nano, 2012, 6, 6930-6935. 